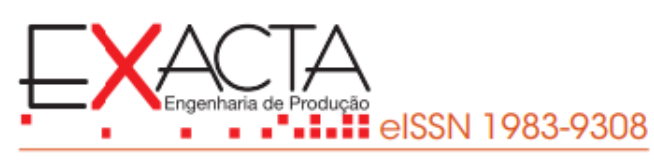

\title{
PRIORIZAÇÃO DAS CATEGORIAS DE INCIDENTES EM UM SETOR DE TI UTILIZANDO O ANALYTIC NETWORK PROCESS
}

\section{PRIORITIZING THE CATEGORIES OF THE INCIDENTS IN A IT SECTOR UTILIZING THE ANALYTIC NETWORK PROCESS}

Versão do autor aceita publicada online: 13 fev. 2020

Publicado online: 12 maio 2021

Como citar esse artigo - American Psychological Association (APA):

Almeida, R. G., Leite, T. B., \& Hernandez, C. T. (2021, abr./jun.). Priorização das categorias de incidentes em um setor de TI utilizando o Analytic Network Process. Exacta, 19(2), 456-476. https://doi.org/10.5585/exactaep.2021.11378.

Submeta seu artigo para este periódico $\beta$

Dados Crossmark 


\section{PRIORIZAÇÃO DAS CATEGORIAS DE INCIDENTES EM UM SETOR DE TI UTILIZANDO O ANALYTIC NETWORK PROCESS}

\section{PRIORITIZING THE CATEGORIES OF THE INCIDENTS IN A IT SECTOR UTILIZING THE ANALYTIC NETWORK PROCESS}

\section{Rafael Guimarães de Almeida ${ }^{1}$ \\ iD Thiago Brum Leite ${ }^{2}$ \\ iD Cecília Toledo Hernandez ${ }^{3}$}

${ }^{1}$ Mestre em Modelagem Computacional em Ciência e Tecnologia

Universidade Federal Fluminense - UFF. rafael_almeida@id.uff.br

2 Mestrando do Programa de Pós-Graduação em Modelagem Computacional em Ciência e Tecnologia Universidade Federal Fluminense - UFF. thiagobrum@id.uff.br

\footnotetext{
${ }^{3}$ Doutora em Engenharia Mecânica Universidade Federal Fluminense - UFF ctoledo@id.uff.br
}

Recebido em: 21 jan. 2019

Aprovado em: 13 fev. 2020
Resumo: O presente artigo propõe uma metodologia de priorização dos principais incidentes atendidos pelo departamento de Tecnologia da Informação (TI) de uma universidade pública. Para isso, utilizou-se as recomendações da Biblioteca Information Technology Infrastructure Library $\left(I T I{ }^{\circledast}\right)$, para identificação e categorização dos principais incidentes, e, posteriormente, o método Analytic Network Process (ANP), por meio do software SuperDecisions, a fim de determinar um ranking de prioridade nos atendimentos a tais incidentes, nos diferentes cenários analisados. Além disso, visando reduzir o número de cenários, compactando cenários similares em apenas um único cenário, através da teoria da compatibilidade, os vetores de decisão obtidos para tais cenários foram avaliados. Os resultados sugeriram, ao departamento de $\mathrm{TI}$, diferentes formas de atuações, de acordo com os rankings gerados para cada cenário, e uma impossibilidade na generalização entre os cenários relacionados às visitas.

Palavras-chave: ITIL ${ }^{\circledR}$. Métodos de apoio a decisão. ANP. SuperDecisions. Compatibilidade.

Abstract: The present article proposes a methodology of prioritizing the main incidents attended by the Information Technology (IT) department of a public university. To that end, the recommendations of Information Technology Infrastructure Library have been used $\left(\mathrm{ITIL}^{\circledR}\right)$, for identification and categorization of main incidents, and, afterwards, the Analytic Network Process (ANP) method, throughout the software SuperDecisions, in order to determine a priority ranking in the assistance to such incidents, in different scenarios. Besides that, aiming to reduce the quantity of scenarios, compacting similar scenarios in only one, through the compatibility theory, the vectors of decision obtained to such scenarios have been evaluated. The results have suggested, to the IT department, different ways of acting, according to the generated rankings to each scenario, and one impossibility in the generalization among scenarios related to visitors.

Keywords: ITIL ${ }^{\circledast}$. Multicriteria decision methods (MCDM). ANP. SuperDecisions. Compatibility. 


\section{Introdução}

A tecnologia não deve ser entendida como um equipamento, produto ou componente, pois estes, apesar de muitas vezes serem resultado da tecnologia, não são a tecnologia em si. Conforme Veraszto, da Silva, Miranda e Simon (2009), a tecnologia é um pacote de informações organizadas, de diferentes tipos (científicas, empíricas...), provenientes de várias fontes (descobertas científicas, patentes, livros, manuais, desenhos...), obtidas através de diferentes métodos (pesquisa, desenvolvimento, cópia, espionagem...) e utilizada na produção de bens e serviços. A tecnologia, portanto, não é um bem tangível que pode ser comprado, mas é um saber que se adquire pela educação teórica e prática, e, principalmente, pela pesquisa tecnológica.

A Tecnologia da Informação (TI), por sua vez, está relacionada aos meios utilizados pelas empresas para impulsionar e incrementar o processo de desenvolvimento da tecnologia com a qual ela trabalha. Este processo é feito através de um Sistema de Informação (SI) que, de acordo com Silva (2001), é um conjunto interdependente composto de Recursos Humanos, Software, Hardware, Redes e Dados, que visam executar atividades de entrada, processamento, armazenamento e controle com o objetivo de transformar recursos de dados em produtos de informação, que poderão ser utilizados para apoiar a tomada de decisão, a coordenação e a análise, geralmente, em configurações organizacionais. Estas relações entre componentes e atividades desenvolvidas em um SI é mostrada na Figura 1.

Figura 1 - Componentes de um Sistema de Informação

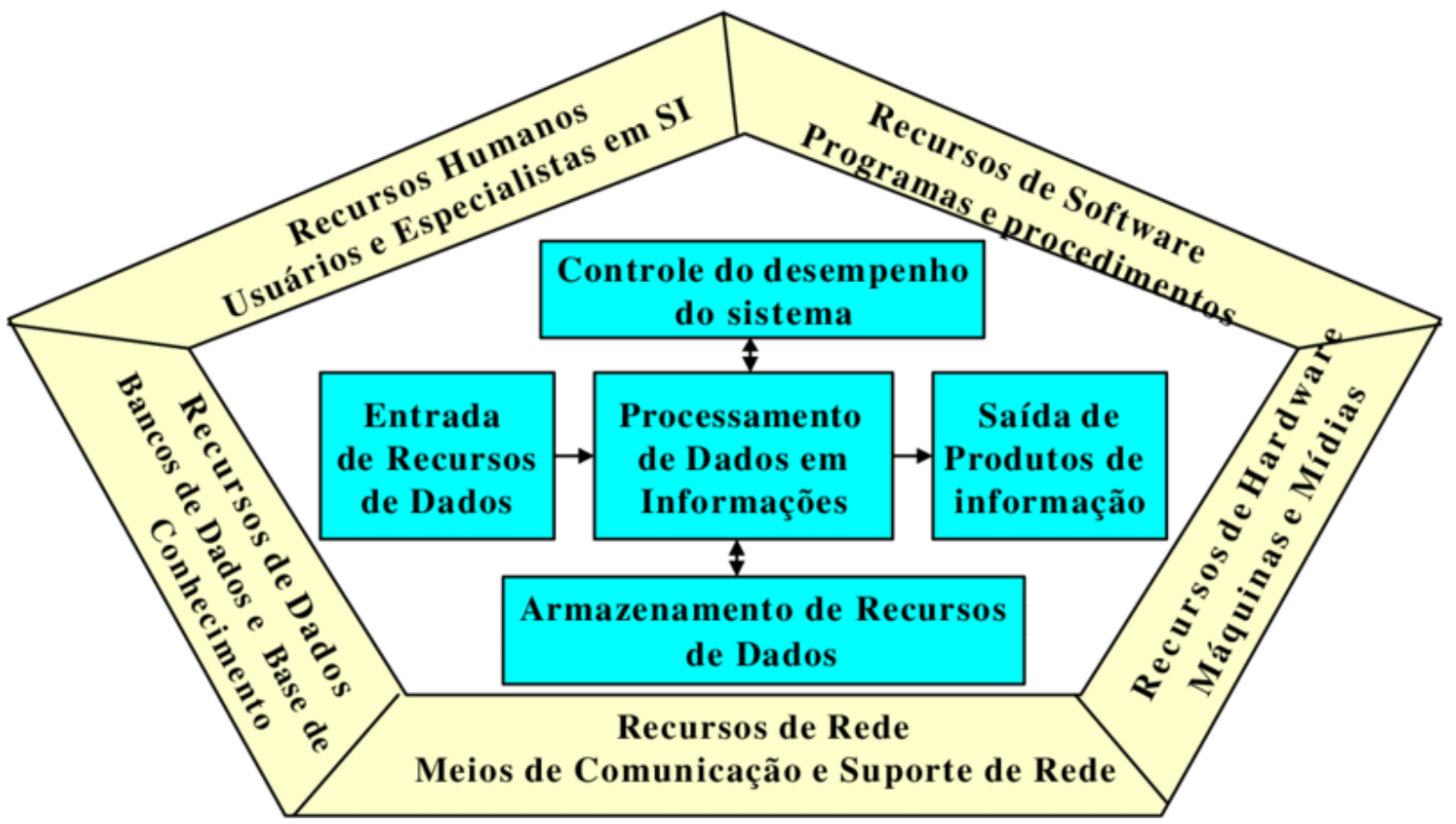

Fonte: Silva (2001). 
Segundo a ISO/IEC 38500 (2015), a Gestão da TI realiza o planejamento, construção, execução e monitoramento dos serviços relacionados a TI de uma empresa, visando modelar e executar processos inerentes à TI. Enquanto que a Governança de TI aborda técnicas que visam avaliar opções estratégicas, dar uma direção para a TI e monitorar os resultados continuamente. É importante compreender que existe uma diferença, apesar de sutil, entre Governança e Gestão de Serviços de TI, pois existem modelos de Governança de TI robustos que tratam especificamente sobre a implementação de cada uma delas, sendo o Control Objectives for Information and Related Technologies (COBIT), responsável pela Governança de $\mathrm{TI}$, e a Information Technology Infrastructure Library (ITIL $\left.{ }^{\circledR}\right)$, responsável pela Gestão de Serviços de TI, podendo ambas serem aplicadas em conjunto ou individualmente, de acordo com as necessidades da empresa.

Com a crescente demanda por serviços de TI, a necessidade de equipes de profissionais que sejam capazes de manter a produtividade, qualidade e efetividade de todo o parque tecnológico das empresas é cada vez maior. Para tanto, estes profissionais têm lançado mão de recursos científicos e tecnológicos para atender as necessidades e expectativas do mercado. E, não poderia ser diferente na área de Ensino, onde esta também tem aprimorado suas atividades, desde a parte administrativa até sua estrutura, através da TI.

A estrutura da universidade analisada no presente estudo, por exemplo, se caracteriza por possuir diversos laboratórios de informática, salas contando com datashows ou retroprojetores, servidores de gerenciamento e segurança de rede, central telefônica, roteadores wireless, entre outros recursos. Neste contexto, torna-se evidente o crescimento da demanda por serviços de TI. Em contrapartida, o quadro de pessoal e os investimentos nesta área não acompanharam este crescimento por um longo período.

Assim, o objetivo deste trabalho é estabelecer as prioridades das categorias de incidentes para diferentes cenários. Isto permitirá gerenciar o processo de atendimento de clientes pelo departamento de TI, priorizando, de acordo com os critérios definidos e os julgamentos obtidos, os atendimentos considerados mais críticos, conforme o cenário analisado.

Partiu-se do trabalho desenvolvido por Bailão (2018), que utilizou conjuntamente a ITIL ${ }^{\circledR}$ em sua Versão 3 (V3), a técnica Delphi e o Analytic Network Process (ANP) para categorizar e priorizar os atendimentos do departamento de TI de uma universidade pública, porém a autora analisou o comportamento das categorias de incidentes em um único cenário.

De acordo com Topin, Borges e Ribeiro (2017), a biblioteca ITIL ${ }^{\circledR}$ oferece uma abordagem sistemática, testada e comprovada, que objetiva oferecer um conjunto de boas práticas de gerenciamento de serviços de TI. Aproximadamente, para Abraham, Reis, Neto e Beneton (2015), metade dos profissionais de $\mathrm{TI}$ estão bem interacionados à $I T I L^{\circledR}$, o que demonstra uma grande aceitação por parte dos mesmos. Nesta perspectiva, a biblioteca ITIL ${ }^{\circledR}$ foi utilizada para o levantamento 
dos critérios e das categorias de incidentes a serem considerados nesta pesquisa. Também foi utilizada a técnica Delphi, como um survey controlado para síntese dos resultados obtidos durante as entrevistas, pois, segundo dos Santos e Pedron (2017), diz respeito a uma modalidade eficiente de obtenção de dados qualitativos, onde reúne-se um grupo de especialistas em determinado assunto, cada um individualmente apresenta suas estimativas a respeito de assuntos específicos para um pesquisador que analisa os dados e, se necessário, faz uma nova entrevista individual ou coletiva com os especialistas e emite um relatório de síntese. Finalmente, o método ANP foi escolhido, devido à estrutura em redes do problema analisado, para gerar o ranking dos incidentes em cada cenário especificado, a fim do departamento de TI tomar conhecimento dos atendimentos que exigem maior atenção.

Atualmente, a equipe do departamento de TI é composta por uma gestora, dois técnicos de manutenção, dois analistas em rede e um analista de sistema. O departamento atende a comunidade externa e a comunidade interna, onde esta última envolve docentes, discentes, técnicos administrativos e terceirizados. E, os serviços prestados a este parque tecnológico englobam atividades, definidas e categorizadas de acordo com o ITIL ${ }^{\circledast}$ e com a técnica Delphi, que são: Hardware, Software, Infraestrutura, Telefonia e Sistemas.

\section{Referencial teórico}

\subsection{0 método ANP e suas características}

O método ANP é um método multicritério discreto desenvolvido por Saaty (1996). Assim como o método Analytic Hierachy Process (AHP), o ANP, generalização do AHP, se configura como uma metodologia para auxílio na determinação de prioridades no que tange à decisões envolvendo múltiplos critérios. Entretanto, conforme Saaty (2005), as diferenças entre ambas metodologias estão vinculadas às relações de dependências que ocorrem no ANP, isto é, enquanto o AHP possui uma ideia geral de hierarquia, o ANP, por sua vez, permite relações de dependência e feedback entre critérios, subcritérios e alternativas, instituindo, assim, uma ideia de rede cujos elementos constitutivos de tal rede interagem entre si, afetando uns aos outros sob determinada perspectiva, o que contraria o Axioma da Independência do AHP.

Nesse sentido, o método ANP seria uma abordagem mais adequada às situações que dispensam a hipótese de independência entre os elementos de um nível superior, inferior ou similar, fornecendo ao decisor uma representação mais realista, visto que, no mundo real, a dependência de critérios não é algo raro de acontecer. E, quanto às aplicações, como afirmam Ossadnik, Schinke e Kaspar (2016), o ANP vem sendo utilizado em diversas problemáticas de decisão envolvendo seleção, avaliação ou alocação em análise de custo-benefício e, ainda, problemas de previsão. Maiores detalhes quanto as 
fundamentações e aplicações do método ANP podem ser encontrados em Saaty (1996), Saaty (2005), Saaty (2013) e Kadoić, Ređep e Divjak (2017)

\subsection{Procedimentos e etapas}

O procedimento analítico do método ANP, segundo Van Horenbeek e Pintelon (2014), inicia-se com a definição dos critérios e subcritérios de controle, que serão utilizados para a comparação dos elementos da rede. Posteriormente, define-se os clusters, que representam grupos de elementos que possuem características similares e relevantes para o problema, e os elementos, que constituem os clusters. Com isso, define-se, então, a rede, estabelecendo as interações entre os clusters. A próxima etapa realiza a comparação par a par com os elementos de um dado cluster para cada um dos elementos dos clusters influenciados por tal componente, utilizando a Escala Fundamental de Saaty, Tabela 1, o que torna possível a construção da Supermatriz Original (sem pesos). Seguidamente, é feita a comparação par a par entre clusters, gerando uma Matriz de Pesos dos Clusters, que será multiplicada a Supermatriz Original a fim de constituir a Supermatriz Ponderada, que será elevada à potência até a sua convergência e formação da Matriz Limite. Finalmente, um ranking de prioridades das alternativas e de clusters é gerado e o processo se encerra com uma análise de sensibilidade dos resultados

Tabela 1 - Escala fundamental de Saaty explicativa

\begin{tabular}{|c|c|c|}
\hline Intensidade de importância & Definição & Explicação \\
\hline 1 & Igual importância. & $\begin{array}{l}\text { As duas atividades contribuem } \\
\text { igualmente para o objetivo. }\end{array}$ \\
\hline 3 & $\begin{array}{l}\text { Moderada importância de um } \\
\text { sobre o outro. }\end{array}$ & $\begin{array}{l}\text { A experiência e a opinião } \\
\text { favorecem levemente uma } \\
\text { atividade sobre a outra. }\end{array}$ \\
\hline 5 & Importância essencial ou forte. & $\begin{array}{l}\text { A experiência e o julgamento } \\
\text { favorecem fortemente uma } \\
\text { atividade sobre a outra. }\end{array}$ \\
\hline 7 & Importância muito forte. & $\begin{array}{l}\text { Uma atividade é fortemente } \\
\text { favorecida e sua dominância } \\
\text { demonstrada na prática. }\end{array}$ \\
\hline 9 & Extrema importância. & $\begin{array}{l}\text { A evidência favorece uma } \\
\text { atividade em detrimento de } \\
\text { outra. }\end{array}$ \\
\hline $2,4,6,8$ & $\begin{array}{l}\text { São valores intermediários entre } \\
\text { os dois julgamentos adjacentes. }\end{array}$ & \\
\hline
\end{tabular}

\subsection{Vantagens e desvantagens do ANP}

A possibilidade do uso de informações/variáveis quantitativas e qualitativas, a versatilidade de combinação com outras técnicas, a baixa complexidade no uso e a validação demonstrada em estudos empíricos, podem ser enumeradas como algumas das vantagens na utilização dos métodos AHP e ANP 
no âmbito de tomada de decisão multicritério. Por outro lado, quando comparado ao método AHP, o ANP se destaca, principalmente, pela possibilidade de lidar com problemas que não respeitam o axioma da independência devido a interações (dependência ou feedback) de seus elementos entre si (alternativas, subcritérios ou critérios), como se vê em Gupta, Singh e Garg (2015). Portanto, não seria impróprio afirmar, como feito por Wicher e Lenort (2014), que o ANP pode atuar em problemáticas de decisão mais compatíveis com a realidade. Entretanto, apesar do ANP ser capaz de lidar sistematicamente com todos os tipos de dependência e feedbacks no sistema de decisão, Khan et al. (2016) apontam que sua desvantagem está no maior número de perguntas, o que pode tornar a aplicação mais complexa e demorada.

\section{4 Índices de compatibilidade entre vetores}

A análise da compatibilidade entre vetores de decisão foi, segundo Garuti (2016), inicialmente proposta e aplicada em 2002 e, em geral, é utilizada para quantificar o nível de similaridade entre vetores analisados, considerando, para isso, uma relação de proximidade entre vetores em questão. Dentre as diversas aplicações desta análise, podemos citar: mensuração do grau de correspondência ou proximidade entre compradores e vendedores; mensuração do nível de consenso de opiniões em ambientes de tomada de decisão em grupo; e, mais recentemente, redução de incertezas e reconhecimento de padrões em diagnósticos na medicina, psiquiatria e psicologia. Maiores detalhes destas aplicações podem ser encontradas, por exemplo, em Garuti e Salomon (2012), Jiang, Xu e Yu (2013) e Garuti (2016). Para efetuar uma análise de compatibilidade, estudos apontam a utilização de três índices de compatibilidade principais, que são: índice $S$, de Saaty; índice $G$, de Garuti; e índice $V$, de Salomon (Guimarães, 2017).

\subsubsection{O índice $S$ de Saaty}

O índice de compatibilidade $S$, proposto por Saaty (2005), foi a primeira medida de compatibilidade entre vetores de prioridades apresentada. Deste modo, considerando dois vetores de prioridades, $x$ e $y$, ambos de dimensão $n$, o índice $S$ é obtido conforme a Equação (1), cujo $e$ representa um vetor com componentes iguais a 1 (também de ordem $n$ ) e $(\cdot)^{T}$ denota a matriz transposta. Cabe mencionar que $A$ e $B$ são matrizes geradas a partir dos componentes dos vetores $x$ e $y\left(a_{i j}=x_{i} / x_{j}\right.$ e $\left.b_{i j}=y_{i} / y_{j}\right)$ e que $A \cdot B$ denota o Produto de Haddamard entre as matrizes, isto é, $a_{i j} \cdot b_{i j}=a_{i j} b_{i j}$.

$$
S=\left(\frac{1}{n^{2}}\right) e^{T} A \cdot B^{T} e
$$


Deste modo, quanto mais o valor de $S$ se aproximar de 1,0, mais próximos os vetores estão entre si, podendo, então, serem considerados compatíveis. Já para $S>1,1$, é sugerido aceitar a hipótese de não compatibilidade. No caso de vetores idênticos, em consoância com Saaty (2005), $S$ deve ser igual a 1,0 .

Salomon (2010), porém, demonstra que não é recomendado utilizar unicamente o índice $S$ para a verificação de compatibilidade entre vetores, visto que o mesmo possui sensibilidade a valores pequenos. Esta sensibilidade, para Salomon (2010), não é com relação ao valor absoluto do componente, mas, sim, com relação a seu valor relativo, ou seja, se algum componente for relativamente pequeno, então o índice de consistência obtido pode indicar incompatibilidade entre vetores compatíveis

\subsubsection{O índice G de Garuti}

Utilizando fundamentos da Álgebra Linear, assim como Saaty (2005), Garuti (2007) propõe uma abordagem alternativa para determinação de indicadores de compatibilidade, através do conceito de produto interno. Considerando dois vetores, segundo o autor, quanto mais o ângulo entre os vetores se aproxima do valor zero e $G$ tende para um, maiores são as possibilidades de compatibilidade entre os mesmos. No contexto dos vetores possuírem maiores quantidades de elementos, Garuti e Salomon (2012) propõem uma tendência para redução do índice $G$.

A Equação (2) apresenta a relação para obtenção do índice de compatibilidade $\mathrm{G}$, onde $x$ e $y$, são dois vetores de prioridades, ambos de dimensão $n$.

$$
G=\frac{1}{n} \sum_{i=1}^{n}\left[\frac{\left(x_{i}+y_{i}\right)}{2} \frac{\min \left(x_{i} ; y_{i}\right)}{\max \left(x_{i} ; y_{i}\right)}\right]
$$

A Tabela 2 apresenta a classificação do índice de compatibilidade $G$ de acordo com os respectivos limites de obtenção:

\begin{tabular}{|c|c|}
\hline Intervalo & Classificação \\
\hline$G \geq 0,900$ & Alta compatibilidade \\
\hline $0,850 \leq G \leq 0,899$ & Quase totalmente compatível \\
\hline $0,750 \leq G \leq 0,849$ & Compatibilidade moderada \\
\hline $0,650 \leq G \leq 0,749$ & Baixo nível de compatibilidade \\
\hline $0,600 \leq G \leq 0,649$ & Muito pouca compatibilidade \\
\hline$G<0,600$ & Totalmente incompatível \\
\hline
\end{tabular}

Fonte: Garuti (2016). 
Saaty e Peniwati (2013) consideraram a utilização do índice $G$, em consequência da sensibilidade presente no índice $S$, quando aplicado em comparações envolvendo valores de prioridades maiores com menores.

\subsubsection{O índice $V$ de Salomon}

De acordo com Salomon (2010), para a obtenção do índice $S$, não se leva em consideração possíveis sensibilidades à componentes, relativamente, pequenos. Tal sensibilidade pode não ser um problema na prática, mas, para um grande número de alternativas, resultados incorretos podem ser gerados, como a incompatibilidade entre vetores que são, em princípio, compatíveis. Assim, visando contornar tal situação, Salomon (2010) propõe um índice de compatibilidade ordinal, de forma similar à relação proposta por Saaty (2005), utilizando, em sua relação, vetores ordinais.

Diante disso, seria possível e recomendável, pelo autor, utilizar os índices $S$, de Saaty, e $V$ em conjunto, pois, o primeiro indicaria uma compatibilidade cardinal, enquanto o segundo indicaria uma compatibilidade ordinal, o que traria uma melhor interpretação de resultados. Assim, conforme Salomon (2010), tanto problemas de escolha quanto problemas de ordenação possuiriam indicadores com resultados diferentes.

Considerando dois vetores de prioridades, $x$ e $y$, ambos de dimensão $n$, e dois vetores ordinais $o$ e $p$, formados pela ordem das prioridades de $x$ e $y$, respectivamente, isto é, $o_{i}=\operatorname{ordem}_{i=1}^{n}\left(x_{i}\right)$ e $p_{i}=\operatorname{ordem}_{i=1}^{n}\left(y_{i}\right)$. Logo, o índice $V$ de Salomon é obtido conforme a Equação (3), cujo $e$ representa um vetor com componentes iguais a 1 (também de ordem $n$ ) e $(\cdot)^{T}$ denota a matriz transposta. Cabe mencionar que as matrizes $C$ e $D$ são geradas a partir dos componentes dos vetores $o$ e $p\left(c_{i j}=o_{i} / o_{j}\right.$ e $d_{i j}=p_{i} / p_{j}$ ) e que $C \cdot D$ denota o Produto de Haddamard entre as matrizes, isto é, $c_{i j} \cdot d_{i j}=c_{i j} d_{i j}$.

$$
V=\left(\frac{1}{n^{2}}\right) e^{T} C \cdot D^{T} e
$$

Ainda segundo Salomon (2010), no que tange o comportamento do índice de compatibilidade ordinal, na hipótese de os vetores de decisão apresentarem a mesma ordem, considera-se a existência de compatibilidade ordinal entre vetores. Como limite para a compatibilidade ordinal, é proposto pelo autor que $V \leq 1,1$. 


\section{Metodologia}

Como anteriormente mencionado, a presente pesquisa se caracteriza por ser uma extensão do trabalho realizado por Bailão (2018). Porém, diferentemente do trabalho inicial, levou-se em consideração seis possíveis cenários alternativos, frequentes no ambiente estudado e com diferentes variáveis externas, que são eles: Banca de Concursos, Inscrição em Disciplinas, Matrícula de Novos Alunos, Visita de Órgãos Externos, Visita da Reitoria e Visita do MEC.

A pesquisa foi empregada em formato de estudo de caso, onde um dos pesquisadores é membro da situação pesquisada. Quanto à sua natureza, se classifica como pesquisa aplicada, porque busca investigar e explicar a solução do problema da pesquisa, através da modelagem das prioridades e simulação em cenários propostos, pois de acordo com Silva e Menezes (2005), tal pesquisa gera conhecimento para aplicações e soluções práticas para o problema estudado. Já de acordo com o objetivo, se classifica como exploratória, que, segundo Gil (2002), são aquelas que visam proporcionar maior familiaridade com o problema, o tornando menos complexo ou constituindo hipóteses.

Seguindo o modelo implementado por Bailão (2018), foram considerados dois importantes critérios considerados pela biblioteca ITIL ${ }^{\circledR}$, são eles: Impacto e Urgência. É importante considerar estes critérios na determinação das prioridades, pois segundo Cestari (2012), configura-se em uma boa prática da $I T I L^{\circledR}$, utilizar a combinação de Impacto e Urgência na determinação de prioridades, onde Impacto refere-se ao número de usuários/clientes e/ou sistemas que serão afetados por um incidente, enquanto Urgência refere-se ao tempo de atendimento máximo dentro do qual o incidente deve ser resolvido.

Como método para coleta de dados, foram realizadas duas entrevistas, direcionadas e respondidas pelos especialistas do departamento de $\mathrm{TI}$, guiadas por um roteiro estruturado, baseado na literatura pesquisada em artigos científicos nacionais e internacionais e em revistas técnicas disponibilizadas pelo foco do estudo. A técnica Delphi foi associada às entrevistas em grupo. Desta forma, foi possível obter um consenso nos julgamentos realizados pelos especialistas da área estudada.

Para a categorização dos incidentes e suas relações de dependência, foram utilizados dados obtidos da primeira entrevista, bem como dados obtidos pela análise dos incidentes registrados no Gestionnaire Libre de Parc Informatique (GLPI), este trata-se do sistema de Gerenciamento de Incidentes de TI utilizado pelos especialistas da universidade em questão. A partir destes dados montouse o modelo da Figura 2. 
Figura 2 - Modelo específico de redes para aplicação do método ANP

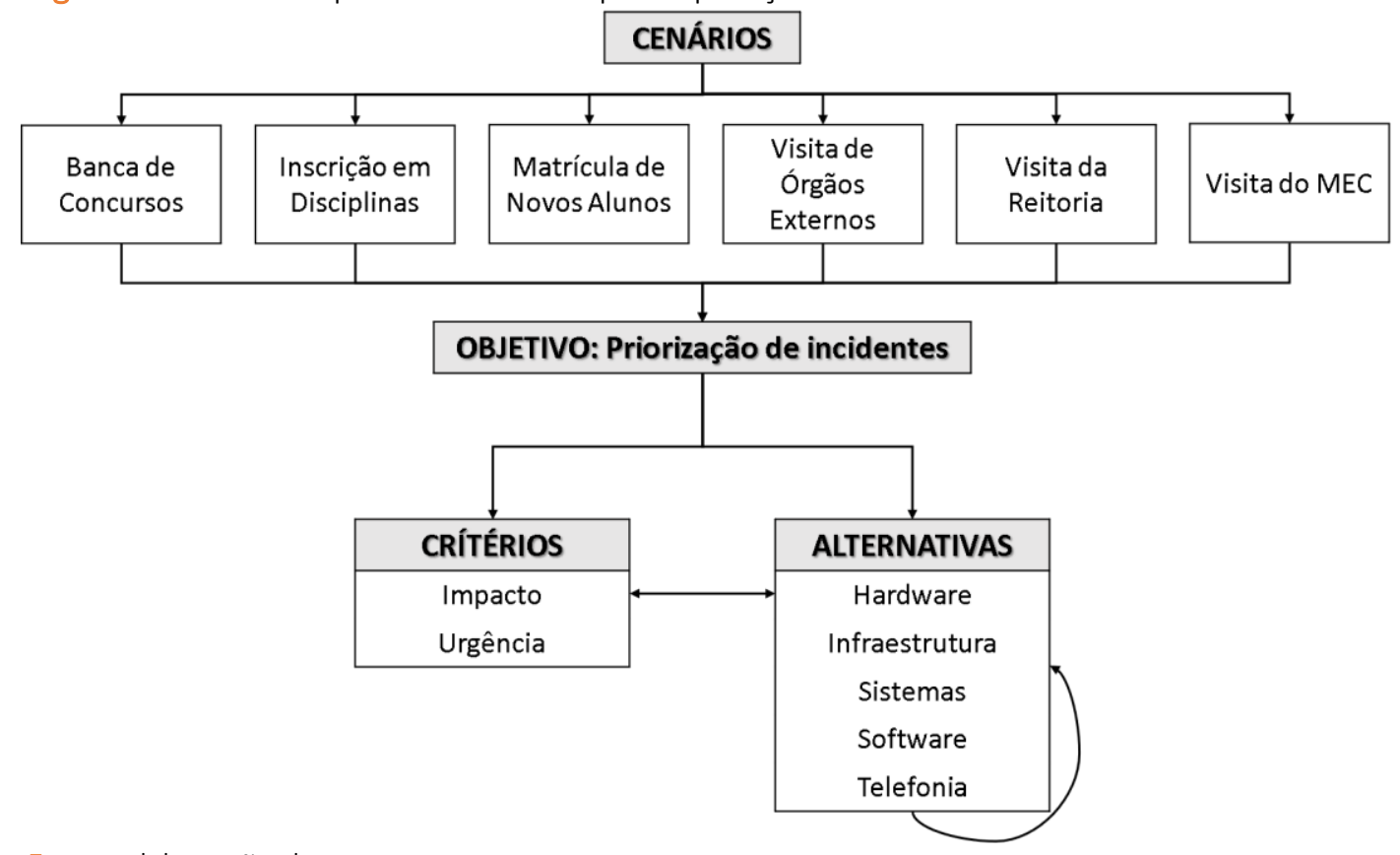

Fonte: Elaboração dos autores.

O modelo, representado pela Figura 2, por sua característica de rede, permite utilizar o método ANP e, consequentemente, estabelecer as prioridades do Impacto e Urgência (chamadas de critérios) e das categorias dos incidentes identificadas (chamadas de alternativas). A aplicação do ANP seguiu os procedimentos e etapas presentes em Van Horenbeek e Pintelon (2014).

Assim, uma segunda entrevista foi realizada e repetida nos seis diferentes cenários, tendo como objetivos a comparação e atribuição dos pesos para cada um dos elementos e clusters das matrizes de alcance global e local, presentes no método ANP. Nestas entrevistas, cada um dos especialistas, utilizando a Escala Fundamental de Saaty (Tabela 1), atribuiu pesos para cada uma das seis categorias em cada um dos seis cenários. Para síntese dos resultados de forma qualitativa, novamente foi utilizada a técnica Delphi.

A aplicação da segunda entrevista para cada um dos seis cenários, separadamente, se deu, pois, cada um representa uma situação diferente que demanda, por parte do departamento de TI, uma atuação também diferente frente aos problemas que surgem. Por exemplo, no cenário Inscrição em Disciplinas, os alunos matriculados nos diversos cursos oferecidos pela universidade, realizam suas inscrições nas disciplinas de forma online e/ou comparecem às Coordenações a fim de se inscreverem nas disciplinas que estiverem aptos e interessados em cursar; já o cenário Visita de Órgãos Externos se caracteriza pelas visitas de autoridades de órgãos governamentais, tais como: Ministro da Educação, Governador do Estado, Prefeito da Cidade, deputados, etc. Há, portanto, atribuições de pesos diferentes para cada cenário. 
O software Superdecisions, disponível em http://superdecisions.com, foi utilizado para a obtenção dos vetores de prioridades, da matriz de peso dos clusters e para verificação da consistência dos julgamentos. Foi utilizado, em conformidade com Saaty e Peniwati (2013), valores próximos de 0,1 para afirmar a existência de consistência nos julgamentos. O cálculo detalhado para o índice de Consistência é demonstrado em Sousa, Jerônimo, de Melo e Aquino (2016), Coutinho, Júnior, Guimarães e Nodari (2016) e Jerônimo, Melo, e Aquino (2016).

Visando examinar potenciais impactos no ranking de prioridades das alternativas, ou seja, verificar o comportamento de uma alternativa prioritária em relação à modificação das restantes, foi realizada uma análise de sensibilidade dos resultados obtidos. Para tal, utilizou-se a função Sensitivity, presente no software Superdecisions, no qual se caracteriza pela fixação de uma variável independente e, de acordo com modificações em seu peso atribuído, permite uma análise gráfica do comportamento de prioridade das alternativas.

A última etapa deste trabalho foi verificar, utilizando índices de compatibilidade, a existência de compatibilidade entre os cenários semelhantes, relacionados à visitas, que são eles: Visita da Reitoria, Visita de Órgãos Externos e Visita do MEC. Caso confirmada a hipótese de compatibilidade entre tais cenários, os mesmos poderão ser agrupados num único cenário generalizado, denominado de Visitas, que irá possuir o mesmo ranking de prioridades para qualquer visita a ser considerada, reduzindo, então, o número de cenários, deste trabalho, para quatro.

\section{Resultados e discussão}

\subsection{Priorização dos Incidentes mediante a aplicação do Analytic Network Process (ANP)}

A priorização dos incidentes e suas categorias é realizada através do método ANP, no qual faz uso de um modelo geral de rede e de uma matriz geral para identificar quais elementos precisam de comparação mediante julgamentos, para cada cenário analisado (Banca de Concursos, Inscrição em Disciplinas, Matrícula de Novos Alunos, Visita da Reitoria, Visita de Órgãos Externos e Visita do MEC). A Figura 3 apresenta o modelo de rede composto pelos critérios (Impacto e Urgência) e alternativas/categorias de incidentes (Software, Hardware, Telefonia, Sistemas, Infraestrutura), junto às suas respectivas relações.

Figura 3 - Modelo de rede Geral para aplicar o ANP

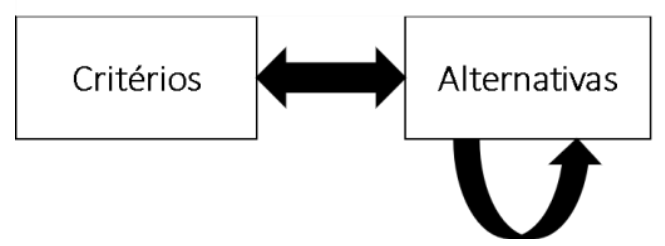

Fonte: Elaboração dos autores. 
A Figura 4 mostra a matriz geral do modelo, considerando que os critérios Impacto e Urgência, neste caso, possuirão a mesma importância, como foi concebido no trabalho de Bailão (2018). Assim, o valor 1 indica a existência de relações entre os elementos, enquanto o valor zero indica que não há relação.

Figura 4 - Matriz Global de relações entre critérios e alternativas

\begin{tabular}{c|cc} 
& Critérios & Alternativas \\
\hline Critérios & 0 & 1 \\
Alternativas & 1 & 1
\end{tabular}

Fonte: Elaboração dos autores.

De posse destas informações, a próxima etapa foi a atribuição de pesos para cada critério em relação às alternativas e entre as alternativas. Isso se deu para todos os seis cenários, separadamente. Para tal, as opiniões dos especialistas, após resultados obtidos utilizando a técnica Delphi, foram lançadas no Software Superdecisions.

As Tabela 3 e 4 apresentam os resultados dos pesos das alternativas/categorias de incidentes em relação ao critério Impacto e Urgência, respectivamente, no cenário Banca de Concursos.

Para as Tabelas 3, 4, 5, 6, 7, 8 e Figura 5, leia-se: $\mathrm{Ha}=$ Hardware; In = Infraestrutura; $\mathrm{Si}=$ Sistemas; So = Software; $\mathrm{Te}=$ Telefonia; $\mathrm{Cr}=$ Coeficiente de consistência .

Tabela 3 - Pesos das alternativas/categorias de incidentes em relação ao critério Impacto

$\begin{array}{llllllll}\text { Impacto } & \text { So } & \text { Ha } & \text { Te } & \text { In } & \text { Si } & \text { Vetor } & \mathrm{Cr} \\ \text { So } & 1 & 1 & 9 & 5 & 5 & 0,379 & \\ \text { Ha } & & 1 & 9 & 5 & 5 & 0,379 & \\ \text { Te } & & & 1 & 1 / 7 & 1 / 7 & 0,026 & 0,102 \\ \text { In } & & & & 1 & 3 & 0,131 & \\ \text { Si } & & & & & 1 & 0,084 & \end{array}$

Fonte: Elaboração dos autores.

Tabela 4 - Pesos das alternativas/categorias de incidentes em relação ao critério Urgência

$\begin{array}{llllllll}\text { Urgência } & \text { So } & \text { Ha } & \text { Te } & \text { In } & \text { Si } & \text { Vetor } & \mathrm{Cr} \\ \text { So } & 1 & 1 & 9 & 3 & 9 & 0,369 & \\ \text { Ha } & & 1 & 9 & 3 & 9 & 0,369 & \\ \text { Te } & & & 1 & 1 / 9 & 1 & 0,032 & 0,044 \\ \text { In } & & & & 1 & 9 & 0,198 & \\ \text { Si } & & & & 1 & 0,032 & \\ \text { Fonte: Elaboração dos autores. } & & & \end{array}$


As alternativas Hardware e Software têm o mesmo peso, pois, neste cenário, o evento acontece localmente, isto é, em uma ou duas salas, nas quais os computadores, ali presentes, são imprescindíveis para a realização do processo; estas duas alternativas são superiores a Infraestrutura e Sistemas, embora também tenham sua participação no processo. A alternativa Telefonia se comporta como a alternativa mais inferior, já que o cenário Banca de Concursos não necessita de telefonia em nenhuma etapa.

As Tabelas 5 e 6 apresentam os resultados das atribuições de peso entre as alternativas/categorias de incidentes que apresentam relações de influência. A alternativa Telefonia (Te) não influencia nenhuma outra alternativa e a alternativa Sistemas (Si) influencia e recebe a influência somente da alternativa Hardware $(\mathrm{Ha})$, e, desta forma, em relação à alternativa Software, o peso é o mesmo.

Tabela 5 - Pesos das alternativas/categorias de incidentes em relação à alternativa Hardware

$\begin{array}{lllll}\text { Elementos } & \text { So } & \text { Si } & \text { Vetor } & \mathrm{Cr} \\ \text { So } & 1 & 1 / 5 & 0,167 & 0,000 \\ \text { Si } & 5 & 1 & 0,833 & \end{array}$

Fonte: Elaboração dos autores.

Tabela 6 - Pesos das alternativas/categorias de incidentes em relação à alternativa Software

$\begin{array}{lllll}\text { Elementos } & \text { Há } & \text { Si } & \text { Vetor } & \mathrm{Cr} \\ \mathrm{Ha} & 1 & 1 & 0,500 & 0,000 \\ \mathrm{Si} & & 1 & 0,500 & \end{array}$

Fonte: Elaboração dos autores.

A Figura 5 apresenta o resultado global das prioridades de todas as categorias. Este resultado foi obtido com a aplicação no software Superdecisions e demonstra, para o cenário Banca de Concurso, que as alternativas Sistemas e Telefonia são, respectivamente, a alternativa de maior e de menor importância.

Figura 5 - Prioridades obtidas para as alternativas/categorias de incidentes no cenário Banca de Concurso

\begin{tabular}{|c|c|c|}
\hline Name & Normalized by Cluster & Limiting \\
\hline $\mathrm{Ha}$ & 0.32754 & 0.242445 \\
\hline $\ln$ & 0.07899 & 0.058469 \\
\hline $\mathrm{Si}$ & 0.36275 & 0.268507 \\
\hline So & 0.21672 & 0.160416 \\
\hline $\mathrm{Te}$ & 0.01399 & 0.010355 \\
\hline Impacto & 0.50000 & $\longdiv { 0 . 1 2 9 9 0 4 }$ \\
\hline Urgência & 0.50000 & 0.129904 \\
\hline
\end{tabular}

Fonte: Elaboração dos autores. 
É importante salientar que quando todas as alternativas/categorias de incidentes foram julgadas, de acordo com cada critério (Impacto e Urgência), as maiores importâncias estavam em Hardware e Software (Tabelas 3 e 4). Porém, o ranking apresentado pela Figura 5 denota Sistemas como a alternativa mais prioritária, seguida por Hardware e Software, respectivamente. Esta mudança na ordem de prioridades é decorrente da relação de influência entre as categorias, característico do ANP, onde, neste caso, as categorias Hardware e Software influenciam a categoria Sistemas (Tabela 5 e 6), gerando alteração em seu peso.

O mesmo procedimento foi realizado para os cinco outros cenários analisados e os resultados obtidos são apresentados nas Tabelas 7 e 8. Todos os coeficientes de consistência encontrados se comportaram dentro dos limites exigidos, isto é, próximos de 0,100.

Tabela 7 - Prioridades obtidas para as alternativas/categorias de incidentes por cenário analisado

\begin{tabular}{lccccc}
\multicolumn{1}{c}{ Cenários } & $\mathrm{Ha}$ & $\mathrm{In}$ & $\mathrm{Si}$ & $\mathrm{So}$ & $\mathrm{Te}$ \\
Banca de Concursos & 0,328 & 0,079 & 0,363 & 0,217 & 0,014 \\
Inscrição em Disciplinas & 0,339 & 0,244 & 0,135 & 0,262 & 0,021 \\
Matrícula de Novos Alunos & 0,215 & 0,345 & 0,226 & 0,195 & 0,019 \\
Visita da Reitoria & 0,305 & 0,206 & 0,223 & 0,167 & 0,099 \\
Visita de Órgãos Externos & 0,124 & 0,496 & 0,186 & 0,100 & 0,094 \\
Visita do MEC & 0,407 & 0,089 & 0,085 & 0,290 & 0,129
\end{tabular}

Fonte: Elaboração dos autores.

Tabela 8 - Ranking de prioridades das alternativas/categorias de incidentes por cenário analisado

\begin{tabular}{lllllll}
\multicolumn{1}{c}{ Cenários } & Ha & In & Si & So & Te \\
Banca de Concursos & 2 & 4 & 1 & 3 & 5 \\
Inscrição em Disciplinas & 1 & 2 & 4 & 3 & 5 \\
Matrícula de Novos Alunos & 3 & 1 & 2 & 4 & 5 \\
Visita da Reitoria & 1 & 3 & 2 & 4 & 5 \\
Visita de Órgãos Externos & 3 & 1 & 2 & 4 & 5 \\
Visita do MEC & 1 & 4 & 5 & 2 & 3
\end{tabular}

Fonte: Elaboração dos autores.

De acordo com a Tabela 8, o Hardware possui a maior prioridade nos cenários de Inscrição em Disciplinas, Visita da Reitoria e Visita do MEC, pois esta categoria se relaciona e influencia todas as outras alternativas, ou seja, se o Hardware apresentar indisponibilidade, tais tarefas são inviabilizadas. A Infraestrutura é a alternativa a ser priorizada em Matrículas de Novos Alunos e Visita de Órgãos Externos, já que a infraestrutura diz respeito ao funcionamento direto da rede interna (LAN) e da comunicação com a rede externa (Internet), onde tais eventos dependem diretamente do funcionamento da internet para que sejam realizados a contento. Já os Sistemas devem ser priorizados no cenário de Banca de Concursos, devido este cenário utilizar ininterruptamente o sistema durante sua execução.

As alternativas Software e Telefonia não devem ser as prioridades principais em nenhum cenário analisado. A Telefonia não é prioridade, pois o funcionamento e manutenção da mesma não é 
de responsabilidade direta da equipe de Tl; enquanto que o Software não é prioridade por não ser essencial para a realização de nenhum dos cenários analisados.

\subsection{Análise de sensibilidade}

Os resultados obtidos sugeriram, em concordância com os julgamentos, uma prioridade à alternativa Sistemas, considerando o cenário Banca de Concursos. Para análise de sensibilidade, foi selecionado a alternativa Hardware como variável independente. Assim, a Tabela 9 apresenta os valores normalizados obtidos, inicialmente, para a alternativa prioritária (Sistemas) e para a variável independente escolhida, junto ao seu respectivo peso.

\begin{tabular}{llr}
\multicolumn{3}{c}{ Tabela 9 - Valores obtidos para } \\
Alternativa & Normalizado & Peso \\
Hardware & 0,327 & 0,242 \\
Sistemas & 0,362 & - \\
\hline Fonte: Elaboração dos autores.
\end{tabular}

Assim, a análise gráfica, possível através da função Sensitivity, permite constatar que para um peso igual ou superior à, aproximadamente, 0,266, a alternativa Hardware muda de posição com a alternativa Sistemas, e se torna a prioritária. Neste sentido, nota-se que uma pequena variação dos julgamentos do decisor irá alterar a ordem de prioridade das alternativas.

O mesmo procedimento foi realizado para todos os outros cenários. Os resultados gerados são apresentados pela Tabela 10, na qual estão presentes, para cada cenário, a Primeira Alternativa Prioritária (PAP), a Segunda Alternativa Prioritária (SAP) e o respectivo Peso atribuído à Segunda Alternativa Prioritária (PSAP), onde foram obtidos a partir dos julgamentos realizados. Além do mais, denota-se juntamente o Peso para/de Troca de Posições (PTP), que provoca mudança de posição da segunda alternativa prioritária com a alternativa situada na primeira posição, obtido de acordo com a análise de sensibilidade.

Tabela 10 - Análise de sensibilidade das alternativas/categorias de incidentes por cenário analisado

\begin{tabular}{lllll}
\multicolumn{1}{c}{ Cenário } & \multicolumn{1}{c}{ PAP } & SAP & PSAP & PTP \\
Banca de Concursos & Sistemas & Hardware & 0,242 & 0,266 \\
Inscrição em Disciplinas & Hardware & Software & 0,177 & 0,224 \\
Matrícula de Novos Alunos & Infraestrutura & Sistemas & 0,145 & 0,211 \\
Visita da Reitoria & Hardware & Sistemas & 0,145 & 0,192 \\
Visita de Órgãos Externos & Infraestrutura & Sistemas & 0,108 & 0,250 \\
Visita do MEC & Hardware & Software & 0,207 & 0,274
\end{tabular}

Nota: a Quanto menor for a diferença entre PTP e PSAP, maior é a sensibilidade.

Fonte: Elaboração dos autores. 
O cenário Banca de Concursos apresentou a maior sensibilidade, visto que, com relação a segunda alternativa, a diferença entre o peso obtido, pelo conjunto de julgamentos, e o peso que a torna prioridade, é a menor dentre todos os cenários. Já o cenário Visita de Órgãos Externos, possui a maior diferença, sendo, portanto, o menos sensível.

\section{3 Índices de compatibilidade para os cenários relacionados às visitas}

Tendo em vista a compactação dos cenários que fazem referência à alguma forma de visitação em apenas um único cenário, serão utilizados os resultados obtidos, demonstrados na Tabela 11, e os índices de Saaty $(S)$, Garuty $(G)$ e Salomon $(V)$, junto suas respectivas classificações conforme seus autores.

Tabela 11 - Tabela de vetores utilizados para testes de compatibilidade

$\begin{array}{llll}\text { Alternativas } & \text { Visita da Reitoria } & \text { Visita de Órgãos Externos } & \text { Visita do MEC } \\ \text { Hardware } & 0,305 & 0,124 & 0,407 \\ \text { Infraestrutura } & 0,206 & 0,496 & 0,089 \\ \text { Sistemas } & 0,223 & 0,186 & 0,085 \\ \text { Software } & 0,167 & 0,100 & 0,290 \\ \text { Telefonia } & 0,099 & 0,094 & 0,129\end{array}$

Fonte: Elaboração dos autores.

Assim, deve-se, então, realizar comparações dois-a-dois entre os vetores para testar a hipótese de compatibilidade entre eles. As Tabelas 12, 13 e 14 demonstram os valores obtidos para os índices de compatibilidade utilizados com suas respectivas classificações. Os índices de compatibilidade foram calculados utilizando as Equações (1), (2) e (3).

Tabela 12 - Índices de compatibilidade entre os cenários Visita da Reitoria e Visita de Órgãos Externos

\begin{tabular}{crl} 
Índices de Compatibilidade & Valor & \multicolumn{1}{c}{ Classificação } \\
$S$ & 1,412 & Incompatível \\
$G$ & 0,576 & Totalmente incompatível \\
$V$ & 1,604 & Incompatível
\end{tabular}

Fonte: Elaboração dos autores. 
Tabela 13 - Índices de compatibilidade entre os cenários Visita da Reitoria e Visita do MEC

\section{Índice de Compatibilidade}

Valor

$S$

G

$V$
1,461 Incompatível

0,608 Muito pouca compatibilidade

1,380 Incompatível

Fonte: Elaboração dos autores.

Tabela 14 - Índices de compatibilidade entre os cenários Visita de Órgãos Externos e Visita do MEC

$\begin{array}{ccl}\text { Índice de Compatibilidade } & \text { Valor } & \text { Classificação } \\ S & 2,996 & \text { Incompatível } \\ G & 0,343 & \text { Totalmente incompatível } \\ V & 2,322 & \text { Incompatível }\end{array}$

Fonte: Elaboração dos autores.

É válido salientar que, pela Tabela 15, é possível analisar previamente a ordem de prioridades obtida. Sendo assim, observa-se que para o cenário Visita da Reitoria, o vetor ordinal das prioridades é dado por $(1,3,2,4,5)^{T}$, enquanto para o cenário de Visita de Órgãos Externos e para Visita do MEC são dados por $(3,1,2,4,5)^{T}$ e $(1,4,5,2,3)^{T}$, respectivamente.

Tabela 15 - Ranking dos cenários relacionados às visitas

$\begin{array}{llll}\text { Alternativas } & \text { Visita da Reitoria } & \text { Visita de Órgãos Externos } & \text { Visita do MEC } \\ \text { Hardware } & 1 & 3 & 1 \\ \text { Infraestrutura } & 3 & 1 & 4 \\ \text { Sistemas } & 2 & 2 & 5 \\ \text { Software } & 4 & 4 & 2 \\ \text { Telefonia } & 5 & 5 & 3\end{array}$

Fonte: Elaboração dos autores.

Diante disso, quando comparadas as prioridades relativas aos cenários Visita da Reitoria e Visita de Órgãos Externos, há uma inversão entre a primeira e a terceira prioridade. Apenas uma prioridade é mantida em uma mesma posição, que é a primeira do ranking, e as demais permutadas, para os cenários Visita da Reitoria e Visita do MEC. Já para os cenários Visita de Órgãos Externos e para Visita do MEC, todas as prioridades se comportaram de maneira diferente. $\mathrm{O}$ grande número de permutações refletiu nos índices de compatibilidade, que demonstraram uma incompatibilidade esperada. 


\section{Considerações finais}

Este trabalho buscou solucionar o problema da priorização dos atendimentos aos incidentes de um departamento de $\mathrm{TI}$, atuante em uma universidade pública, em seis recorrentes cenários. Isto se deu com base na combinação de três diferentes abordagens: biblioteca ITIL ${ }^{\circledR}$, para a determinação dos critérios e das categorias de incidentes desta problemática; técnica Delphi, para a síntese dos resultados gerados nas entrevistas em grupo; método ANP, para a obtenção de um ranking de prioridades dos incidentes considerados mais críticos para cada cenário. Por último, uma análise de compatibilidade foi executada com o intuito de reduzir o número de cenários semelhantes e, consequentemente, o número de situações a serem enfrentadas pelo departamento de TI. Cabe mencionar que esta integração de ferramentas e este uso dos índices de compatibilidade não são comuns na literatura, o que fornece uma contribuição do ponto de vista teórico.

Do ponto de vista prático, foi possível sugerir diferentes atuações do departamento de TI frente aos incidentes, já que diferentes rankings de alternativas foram obtidos para cada cenário, exceto quando são observados os cenários Matrícula de Novos Alunos e Visitas de Órgãos Externos, onde todas as prioridades se comportaram da mesma maneira. Mas, se considerada apenas a alternativa mais prioritária, os cenários Inscrição em Disciplinas, Visita da Reitoria e Visita do MEC poderão ser atendidos da mesma maneira. E, ainda do ponto de vista prático, também foi possível rejeitar a hipótese de generalização dos cenários relacionados a visitas, em virtude de todos os três índices de compatibilidade utilizados sugerirem uma incompatibilidade entre os cenários. Sendo assim, cada cenário se torna particular, devendo ser analisado isoladamente.

\section{Referências}

Abraham, E. R., Reis, J. G., Neto, M. M., \& Beneton, E. J. (2015). Utilização do ITIL ${ }^{\circledR}$ V3 no Brasil: uma verificação da aplicação do domínio "Estratégia de Serviços" entre os profissionais de Tecnologia da Informação. Exacta.

Bailão, F. S. (2018). Implementação do Processo de Gerenciamento de Incidentes: um estudo de caso em um departamento de TI de uma universidade pública. Dissertação de Mestrado apresentada ao Programa de Pós-Graduação em Administração, Instituto de Ciências Humanas e Sociais, Universidade Federal Fluminense. Volta Redonda, RJ.

Cestari, F. F. (2012). ITIL V3 Fundamentos. Rio de Janeiro: RNP/ESR.

Coutinho, J. d., Júnior, F. R., Guimarães, L. G., \& Nodari, C. H. (2016). Barreiras na produção de canade-açúcar no estado da Paraíba (PB). Exacta-EP, 14, 2, 139-336. São Paulo.

dos Santos, T. A., \& Pedron, C. D. (2017). Método delphi aplicado em pesquisas de gestão de projetos: uma perspectiva além do consenso. Management, Iberoamerican Journal of Project, 10(1), 60-80. 
Garuti, C. (2007). "Measuring Compatibility (Closeness) In Weighted Environments: When Close Really Means Close?." . 9th International Symposium on the Analytic Hierarchy Process.

Garuti, C. E. (2016). New advances of the compatibility index "G" in weighted environments. International Journal of the Analytic Hierarchy Process, 8(3).

Garuti, C., \& Salomon, V. A. (2012). "Compatibility indices between priority vectors.". International Journal of the Analytic Hierarchy Process 4.2 , 152-160.

Gil, A. C. (2002). Metodologia científica. 3. São Paulo.

Guimarães, J. L. (2017). Análise multicritério de indicadores da logística reversa na indústria de calçados de Juazeiro do Norte. Tese de doutorado. Guaratinguetá - SP .

Gupta, A. K., Singh, O., \& Garg, R. (2015). Analytic Network Process (ANP): An approach for supplier selection in an automobile organization. European Journal of Advances in Engineering and Technology, 2(9), 83-89.

ISO/IEC 38500. (2015). Information technology -- Governance of IT for the organization - International Organization for Standardization. Fonte: https://www.iso.org/obp/ui/\#iso:std:iso-iec:38500:ed2:v1:en.

Jerônimo, T. B., Melo, F. J., \& Aquino, J. T. (2016). Análise da implementação do modelo multicritério de decisão: como o gestor observa a importância da decisão racional. Exacta - EP, 14, 2, 319-334. São Paulo.

Jiang, Y., Xu, Z., \& Yu, X. (2013). Compatibility measures and consensus models for group decision making with intuitionistic multiplicative preference relations. Applied Soft Computing, 13(4), 20752086.

Kadoić, N., Ređep, N. B., \& Divjak, B. (2017). Decision making with the analytic network process. In The 14th International Symposium on Operational Research. Slovenia.

Khan, J. A., Izaz-ur-Rehman, Khan, S. P., Afzal, W., Qasim, I., \& Khan, Y. H. (2016). An Evaluation of Requirement Prioritization Techniques with ANP. International Journal of Advanced Computer Science and Applications, 7(7), 419-429.

Ossadnik, W., Schinke, S., \& Kaspar, R. H. (2016). Group aggregation techniques for analytic hierarchy process and analytic network process: a comparative analysis. Group Decision and Negotiation, 25(2), 421-457.

Saaty, T. L. (1996). Decision making with dependence and feedback: The analytic network process. Vol. 4922. RWS Publ.

Saaty, T. L. (2005). Theory and applications of the analytic network process: decision making with benefits, opportunities, costs, and risks. RWS publications.

Saaty, T. L. (2008). "Decision making with the analytic hierarchy process.". International journal of services sciences 1.1, 83-98.

Saaty, T. L., \& Peniwati, K. (2013). Group decision making: drawing out and reconciling differences. RWS publications. 
Salomon, V. A. (2010). Contribuições para validação de tomada de decisão com múltiplos critérios. Guaratingueta, São Paulo, Brasil.

Silva, E. L., \& Menezes, E. M. (2005). Metodologia da pesquisa e elaboração de dissertação. 4. ed., 138. Rev. atual. Florianópolis: UFSC.

Silva, R. (2001). Decisão e Informação na Indústria. Transinformação.

Sousa, J. V., Jerônimo, T. d., de Melo, F. J., \& de Aquino, J. T. (2016). Uso do AHP para identificação de perdas da qualidade em empresas de manufatura: um estudo de caso. Exacta-EP, 15, 1, 89-100. São Paulo.

Topin, L., Borges, E., \& Ribeiro, L. (2017). Uma abordagem para adequação de serviços baseada em práticas da ITIL. Anais do XIII Simpósio Brasileiro de Sistemas de Informação, 579-584.

Van Horenbeek, A., \& Pintelon, L. (2014). Development of a maintenance performance measurement framework-using the analytic network process (ANP) for maintenance performance indicator selection. Omega, 42(1), 33-46.

Veraszto, E. V., da Silva, D., Miranda, N. A., \& Simon, F. (2009). Tecnologia: buscando uma definição para o conceito. Prisma.com, 19-46.

Wicher, P., \& Lenort, R. (2014). Comparison of AHP and ANP methods for resilience measurement in supply chains. METAL 2014: 23rd International Conference on Metallurgy and Materials., 19-46. 